\title{
Bronchoscopy in COVID-19 patients: When, how and why. Experience in clinical practice
}

\author{
Carmine Guarino', Cristiano Cesaro ${ }^{1}$, Giuseppe Fiorentino ${ }^{2}$, Francesco Rossi ${ }^{1}$, \\ Benedetto Maria Polverino1, Fiorentino Fragranza ${ }^{3}$, Luca Monastra ${ }^{3}$, Patrizia Murino ${ }^{4}$, Enzo Zamparelli ${ }^{4}$, \\ Giuseppe La Cerra ${ }^{1}$ \\ ${ }^{1}$ Unit of Bronchology, Monaldi Hospital, Azienda Ospedaliera dei Colli, Naples; ${ }^{2}$ Units of Subintensive Care Therapy, \\ Corps G, Cotugno Hospital, Azienda Ospedaliera dei Colli, Naples; ${ }^{3}$ Unit of Anesthesia and Resuscitation, Cotugno \\ Hospital, Azienda Ospedaliera dei Colli, Naples; ${ }^{4}$ Unit of Anesthesia and Resuscitation, Monaldi Hospital, Azienda \\ Ospedaliera dei Colli, Naples, Italy
}

\begin{abstract}
Severe Acute Respiratory Syndrome due to Coronavirus-19 (SARS-CoV-2) is caused by combined alveolar-capillary lung dam-
\end{abstract}

\author{
Correspondence: Carmine Guarino, Unit of Bronchology, Monaldi \\ Hospital, Azienda Ospedaliera dei Colli-Napoli, Via Leonardo Bianchi \\ snc, Naples, Italy. \\ Tel./Fax: +39.081.7062828 \\ Mobile: +39.338 .3264167 . \\ E-mail: carmine.guarino@ospedalideicolli.it
}

Keywords: Bronchoscopy; COVID-19; bronchoalveolar lavage; disposable bronchoscope.

Author's contributions: CC, GLC, FR, PM, IV, LM, EZ, were personally involved in the execution of the described procedures; $\mathrm{CG}, \mathrm{CC}$, GF, FR, BMP, FF, contributed to data collection and on the manuscript revision; $\mathrm{CC}$ and B.M.P. contributed to study design, interpretation and manuscript revision. All the authors had full access to the data and take responsibility for data integrity, collection, accuracy and description. All the authors have read and approved the final version of the manuscript and agreed to be accountable for all aspects of the work

Ethics approval: All procedures performed and described in this article were carried out in accordance with international guidelines and in accordance with the ethical standards of the Institutional Research Committee and the 1964 declaration of Helsinki and its later amendment.

Informed Consent: Obtained from all patients or their guardians at hospitalization.

Conflict of interest: All the authors declare no conflict of interest with this manuscript.

Funding: All the authors declare having not benefited from any source of funding for this work.

Received for publication: 28 December 2020.

Accepted for publication: 8 February 2021.

${ }^{\circ}$ Copyright: the Author(s), 2021

Licensee PAGEPress, Italy

Monaldi Archives for Chest Disease 2021; 91:1744

doi: 10.4081/monaldi.2021.1744

This article is distributed under the terms of the Creative Commons Attribution Noncommercial License (by-nc 4.0) which permits any noncommercial use, distribution, and reproduction in any medium, provided the original author(s) and source are credited. age, with bilateral pneumonia and thrombosis, which often causes respiratory failure. Proper COVID-19 management requires high skills in airway control and the need to perform aerosol-generating procedures such as bronchoscopy, which can increase the possibility of virus spreading among healthcare professionals. In an epidemiologically delicate moment, the multidisciplinary decision on "WHEN, HOW and WHY" to perform bronchoscopies minimizing the risk of COVID-19 transmission, represented a great challenge for all specialists engaged in bronchoscopic procedures. In this work authors want to share all technical aspects of 87 videobronchoscopies performed in confirmed or suspected COVID-19 patients, from $3^{\text {rd }}$ to $6^{\text {th }}$ January 2020 , describing the reason, the organizational and operational model and patients characteristics. Was also evaluated the impact of high-risk procedures such as bronchoscopy on the personnel involved. The disclosure of all technical details, represents, in the opinion of the authors, an important contribution, capable of providing support to all physicians engaged in bronchoscopy procedures in confirmed or suspected COVID-19 patients.

\section{Introduction}

As the COVID-19 pandemic spreads around the world, health systems and health professionals struggle to find a balance between providing medical care to those in need by limiting the spread of the disease and exposure to patients and staff. Severe acute respiratory syndrome (SARS-CoV-2) represented around the world a major challenge for pulmonologists and for all specialists engaged in the execution of bronchoscopies. The primary route of transmission of COVID-19 is the upper respiratory tract [1]. The novel coronavirus has a high human-to-human transmission capacity through aerosolized droplets, with relevance among healthcare professionals who are exposed to a greater risk of infection than the general population due to direct contact with patient secretions [2,3]. Procedures such as orotracheal intubation and bronchoscopy, generating aerosols, increase the possibility of virus spreading and are considered to be at higher risk for the transmission of COVID-19 $[2,3]$. Due to the high risk of COVID-19 pandemic contagion, many healthcare facilities around the world have changed their organizational plans related to bronchoscopy and many scientific associations such as AABIP, Center for Disease Control, Chinese Thoracic Society, A.I.P.O. recommended the maximum limitation of bronchoscopic procedures due to the high risk of contagion [4-7]. Therefore, in an epidemiologically delicate moment, the multidisciplinary decision on "WHEN, HOW and WHY" to perform bron- 
choscopies, minimizing the risk of COVID19 transmission, guaranteeing the necessary patient assistance, represented a great challenge for all specialists engaged in bronchoscopic procedures.

\section{Materials and Methods}

In this work Authors report the experience acquired on "WHEN, WHY and HOW" 87 videobronchoscopies were performed in patients with suspected or confirmed COVID-19, from 03/01/2020 to $06 / 01 / 2020$ using a single-use bronchoscope $\left(\mathrm{Ambu}^{\circledR} \mathrm{aScope}^{\mathrm{TM}}\right.$ ) and with a bronchoscopy setting for COVID-19 patients. All procedures were performed in a single center "Azienda Ospedaliera dei Colli", Cotugno and Monaldi hospitals by a multidisciplinary team made up of pulmonologists and anesthetists and under the supervision of infectious disease specialists. During the most dramatic phase of the novel coronavirus health crisis in Italy, 79 videobronchoscopies have been performed in confirmed COVID-19 patients and 8 videobronchoscopies in patients with clinical and radiological suspect of COVID-19. The informed consent collection took place in all non-intubated patients before the procedure while in intubated patients the procedure was performed urgently.

\section{Results}

The multidisciplinary organizational and operational model in intensive and sub-intensive respiratory care has reported the following results in this clinical experience during the COVID-19 pandemic: all the 87 bronchoscopies were subdivided into 9 groups (Table 1) based on procedure reason and patients characteristics. In particular, the attention has been focused on WHEN, the clinical moment of the disease evolution in which the bronchoscopies were performed; WHY the reason for which bronchoscopies were carried out; and especially on HOW, the technical details and the operational and organizational model with which bronchoscopies have been performed in COVID-19 positive and suspected patients.

\section{WHEN to perform bronchoscopy in confirmed or suspected COVID-19 patient?}

Bronchoscopy is technically possible in all clinical stages of COVID-19. In the initial moment of the disease, bronchoscopy is indicated and plays an important role for diagnosis thanks to bronchoalveolar lavage (BAL) only if the upper airway samples (rhinopharyngeal swabs) are negative and there is another hypothesized infectious diagnosis that would significantly change the clinical management [8]. Nasopharyngeal swab is usually the most frequently used collection method to obtain COVID-19 confirmation [8]. Rhino-pharyngeal specimens may not guarantee adequate sensitivity in diagnosing early-stage infection, in these cases, bronchoscopy with bronchoalveolar lavage (BAL) must be performed to confirm or deny the diagnosis [1]. It should be noted that, regardless of how accurate and rapid the laboratory analysis methods are, diagnosis of viral pneumonia, requires the collection of the samples in an adequate way and above all in the right way and time [1]. It therefore follows that the concept of WHEN to perform bronchoscopy represents an essential criterion to obtain the diagnosis. In this clinical experience, 8 video bronchoscopies (Table1) were performed to obtain the diagnosis of COVID19 through the bronchoalveolar lavage (BAL), in 8 intubated patients with $\geq 2$ negative nasopharyngeal swabs and a clinical and radiological suspect of COVID-19. Bronchoalveolar lavage (BAL) confirmed the suspicion of COVID-19 in 4 out of these 8 patients.

\section{WHY to perform bronchoscopy in confirmed or suspected COVID19 patient?}

The indications for bronchoscopy in confirmed COVID19 patients, as reported in Table 1, were:

- $\quad 1^{\text {st }}$ group of 30 COVID-19 intubated patients, for the rapid increase of lung resistance in order to evaluate tracheobronchial canalization for suspected obstruction from secretions or clots. In these cases, at the high-resolution chest CT scan (HRCT), "as shown in Figure1A" the most frequent radiological findings were, widespread ground glass areas and bilateral thickening with prevalence at the lung bases and in the sub pleural regions. The most frequent finding during bronchoscopy was an atrophic mucosa with fluid secretions (Figure1 B,C). In almost all of these cases the reason for the sudden increase in lung resistance was not linked to an airways obstruction but to the presence of pulmonary thromboembolism which was subsequently confirmed in 23 out of the 30 intubated patients examined by subsequently performed chest angio-CT scan.

- $\quad 2^{\text {nd }}$ group of 17 COVID-19intubated patients, bronchoscopy was performed on suspicion of alveolar hemorrhage due to evidence of live blood in the orotracheal tube. The high-resolution

Table 1. The 87 bronchoscopies in confirmed/suspected COVID-19 patients.

\begin{tabular}{lll}
\hline Group & Patients' characteristics & Bronchoscopy reason \\
\hline $1^{\text {st }}$ group & 30 COVID-19 confirmed intubated patients & Lung resistance increase \\
\hline $2^{\text {nd }}$ group & 17 COVID-19 confirmed intubated patients & Suspected alveolar hemorrhage \\
$3^{\text {rd }}$ group & 9 COVID-19 confirmed intubated patients & Reposition of the tracheal tube \\
\hline $4^{\text {th }}$ group & 10 COVID-19 confirmed intubated patients & Endoscopic tracheotomy assistance \\
$5^{\text {th }}$ group & 3 COVID-19 confirmed sedated patients & Difficult intubation assistance \\
\hline $6^{\text {th }}$ group & 2 COVID-19 confirmed intubated patients & Suspected ab ingestis pneumonia \\
$7^{\text {th }}$ group & 1 COVID-19 confirmed intubated patient & Suspected tracheal injury \\
\hline $8^{\text {th }}$ group & 7 covid-19 confirmed patient in NIV & Suspected obstruction by secretions or clots \\
$9^{\text {th }}$ group & 8 COVID-19 suspected intubated patients & Detection of coronavirus-19 in BAL \\
\hline
\end{tabular}


chest CT scan (HRCT) showed rough consolidations in the basal lung regions and widespread multiple and ground glass areas (Figure 2A). From the endoscopic point of view, in all patients of this group, secretions mixed with live blood and clots were found (Figure 2 B,C). Moreover the presence of a markedly hyperemic mucosa was observed, often with superficialization of the submucosal vessels with telangiectasic tracts, in some cases, spontaneously bleeding. Bronchoscopies in this group of patients had a predominantly disobstructive purpose. Authors report that these clinical, radiological and endoscopic evidences have often been associated with a poor prognosis; in fact in 13 out of this 17 patients, bronchoscopy confirmed bleeding from alveolar damage, with subsequent patient exitus. This experience showed that the presence of alveolar hemorrhages in COVID-19 intubated patients has also been associated with anticoagulant and antithrombotic therapies to which these patients were subjected.

- $\quad 3^{\text {rd }}$ group of 9 COVID-19 intubated patients. Bronchoscopy was performed for the repositioning of the orotracheal tube under endoscopic control. COVID-19 patients, intubated, hospitalized in intensive care COVID19 units, often undergo pro- cedures, such as pronation, which involve total mobilization of the whole person. In these cases, the loss of position of the tracheal tube is possible and bronchoscopy is essential to evaluate the correct positioning of the orotracheal tube. In all these 9 patients bronchoscopy revealed the loss of position of the orotracheal tube and allowed its correct repositioning under endoscopic guidance.

- $4^{\text {th }}$ group of 10 COVID-19 intubated patients. Bronchoscopy was performed to assist tracheotomy, in order to allow the correct insertion of the tracheotomy cannula. Severe respiratory failure secondary to COVID19 requires prolonged intubation, which often can make tracheotomy necessary [9]. In all 10 patients of this group, bronchoscopy allowed a correct tracheostomy and a precise insertion of the tracheal cannula.

- $\quad 5^{\text {th }}$ group of 3 COVID-19 intubated patients. Bronchoscopy was performed in the 3 patients for assistance at difficult intubation. The bronchoscope was used urgently as a guide for the passage of the orotracheal tube through the vocal cords. The 3 patients of this group had a neck anatomical conformation that made the traditional intubation difficult. In all 3 patients, the intubation under bronchoscopic guidance was successful.
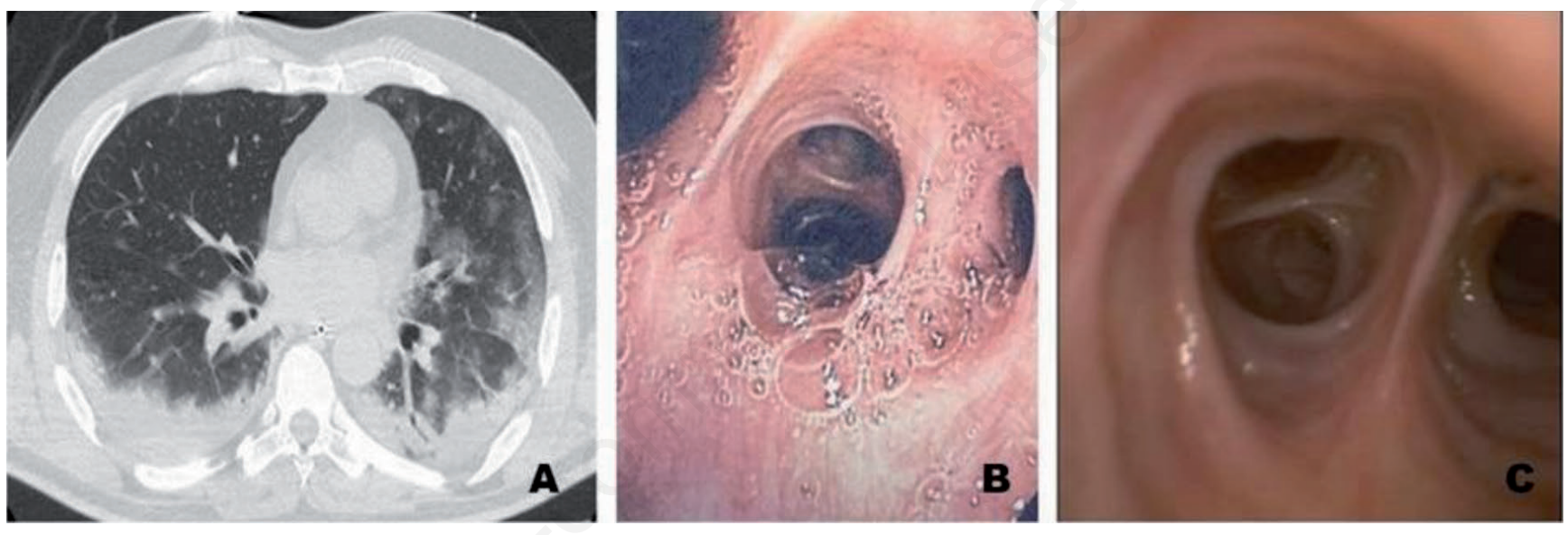

Figure 1. A) Radiological appearance of diffuse areas of ground glass and bilateral thickening with prevalence at the pulmonary bases and in the sub-pleural regions. B,C) Endoscopic aspect of an atrophic mucosa with a prevalence of fluid secretions.
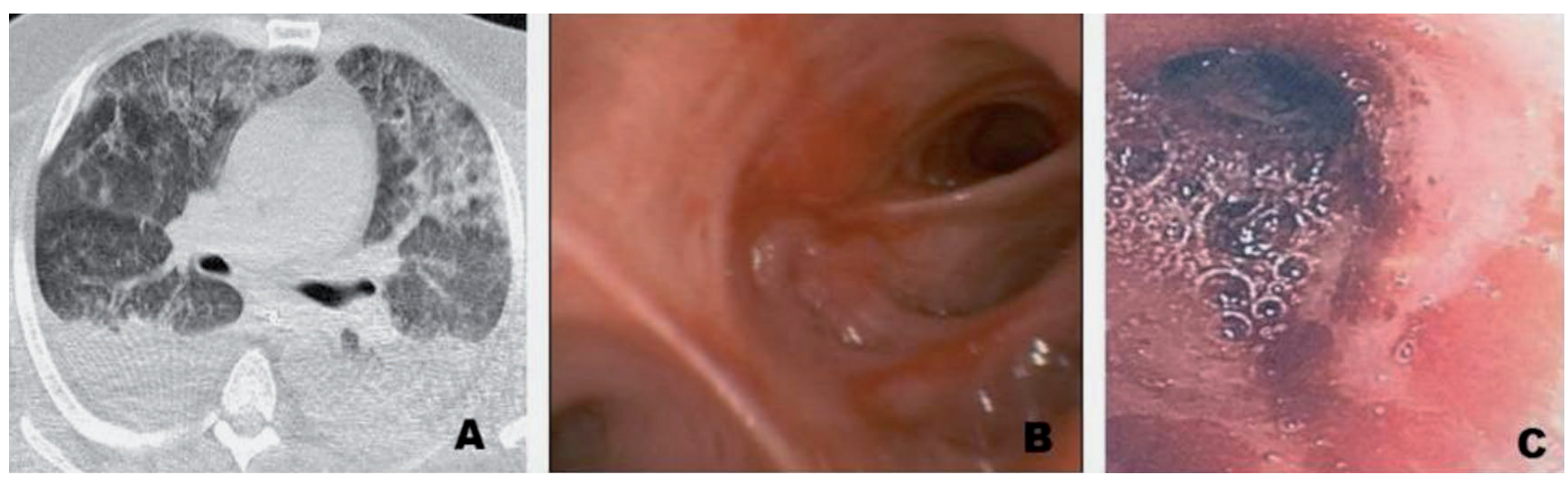

Figure 2. A) High resolution chest CT scan highlights large lung consolidations at the bases mixed with widespread ground glass areas. B,C) Endoscopic pictures of markedly hyperemic mucosa, with superficialization of submucosa vessels and presence of secretions mixed with live blood. 
- $\quad 6^{\text {th }}$ group of 2 COVID-19 intubated patients. Bronchoscopy was performed with the suspicion of ab-ingestis pneumonia. In none of the two cases, bronchoscopy confirmed the diagnostic suspicion.

- $7^{\text {th }}$ group of 1 COVID-19 intubated patient for suspected tracheal injury. On neck and chest CT scan, the patient presented evidence of pneumomediastinum and a radiological suspicion of a tracheal injury. Bronchoscopy revealed no tracheal lesions.

- $\mathbf{8}^{\text {th }}$ group of 7 COVID-19 patients with severe respiratory insufficiency $(\mathrm{PF}<150)$, treated with non-invasive mechanical ventilation (NIV), underwent bronchoscopy for clinical and radiological suspicion of airway obstruction from secretions or clots. In none of the 7 patients of this group, bronchoscopy showed the presence of the suspected airways obstruction. In 5 out of these 7 patients treated in NIV, bronchoscopy had to be stopped due to desaturation ( $\mathrm{SPO} 2<60 \%$ ) and subsequently the 5 patients had to be intubated. The clinical experience gained, in accordance with the recommendations of scientific societies, allows to affirm that bronchoscopy should not be performed in COVID-19 patients with severe respiratory insufficiency subjected to non-invasive mechanical ventilation, due to the high risk of rapid worsening of respiratory symptoms which in many cases can determine or accelerate the need for intubation as well as in order to avoid the dispersion of infected aerosolized particles, which significantly increases the risk of infection [5-8].

- $\quad 9^{\text {th }}$ group of 8 suspected COVID-19 patients were hospitalized in isolation for respiratory failure and bilateral pneumonia experiencing a rapid clinical and respiratory deterioration and have been subsequently intubated. In all 8 patients the highresolution chest CT scan (HRCT) showed thickening and diffuse ground glass areas with bilateral lung involvement. All 8 patients were previously subjected $\geq 2$ nasopharyngeal swabs for coronavirus19 search, with negative results. In all 8 patients, bronchoscopy with bronchoalveolar lavage was required after intubation in order to obtain the COVID-19 diagnosis. In 4 out of 8 patients of this group, bronchoalveolar lavage confirmed the suspicion of COVID-19, and the 4 patients were transferred and managed in a COVID-19 intensive Care Unit. In 2 patients of this group the bronchoalveolar lavage revealed the presence of legionella infection, and in 2 other patients bronchoalveolar lavage showed the presence of fungal infection.

\section{HOW to perform bronchoscopy in COVID-19 patients?}

Seventy-two videobronchoscopies were performed in an emergency regime, in COVID19 intubated patients, in COVID-19 intensive care units, moreover 7 Bronchoscopieshave been practiced in COVID-19 patients treated with non-invasive mechanical ventilation (NIV). Eight videobronchoscopies were performed in suspected COVID-19 intubated patients procedurally managed as if they were positive in order to obtain the diagnosis. Bronchoscopies in suspected or confirmed COVID-19 patients, were processed by a doctor and a nurse using a single-use disposable bronchoscope $\left(\mathrm{Ambu}^{\circledR} \mathrm{aScope} \mathrm{TM}^{\mathrm{TM}}\right)$, with dedicated monitor (Figure 3), in single intensive care rooms, strictly in negative pressure, with depressurization flow $(\Delta$ of $10 \mathrm{~Pa})$ and unidirectional air exchange.

Preparation for videobronchoscopy consists of the dressing phase (Table 2) performed in a room outside the isolation room, performing the procedure in front of another trained operator in order to avoid mistakes. The dressing procedure, in the experience

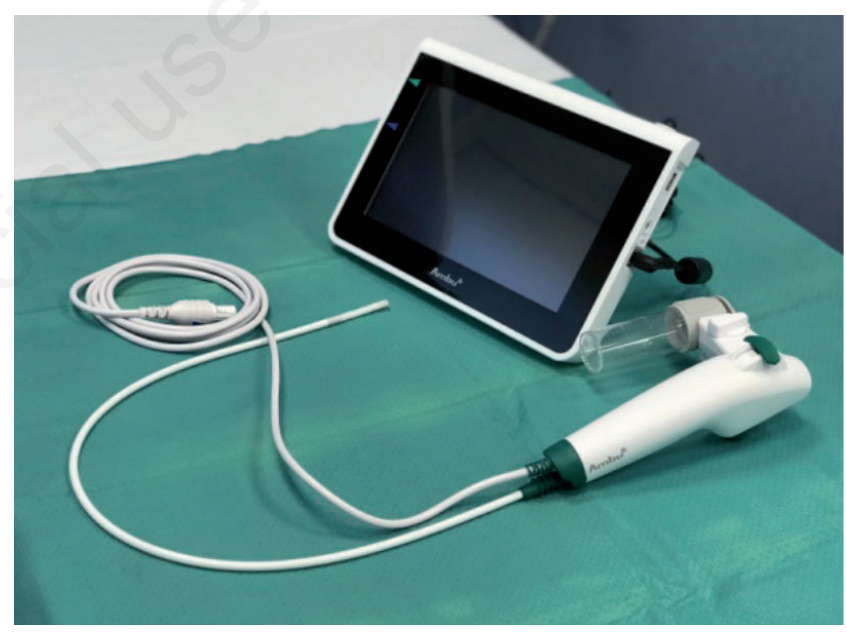

Figure 3. Disposable single-use bronchoscope, with dedicated monitor.

Table 2. Summary of the 11 phases of the dressing and undressing procedure.

\begin{tabular}{|c|c|}
\hline Dressing procedure & Undressing procedure \\
\hline 1) Remove jewelry and personal items and wear disposable uniform & 1) Hands and suit decontamination by spraying sodium hypochlorite solution \\
\hline 2) Check integrity of personal protective equipment & 2) Removing the first pair of gloves \\
\hline 3) Practice hand hygiene (30 seconds) with alcoholic solution or soap and water & 3) Removing the protective screen \\
\hline 4) Wear shoe covers, headgear and the first pair of gloves & 4) Removing suit hood by sliding it back pulling it from the rear end \\
\hline 5) Wear the polypropylene water-repellent suit (excluding hood) & 5) Open suit zipper making it slide until discover shoulders. \\
\hline 6) Wear FFP2/FFP3 mask making it adhere well to face or filtered total-face mask & 6) Free arms from the inside of the suit \\
\hline 7) Wear safety goggles & 7) Complete suit and shoes covers extraction \\
\hline 8) Wear suit hood making it adhere completely to the face & 8) Hands decontamination with sodium hypochlorite \\
\hline 9) Sealing suit zipper along its entire length up to the neck. & 9) Protective glasses extraction and second pair of gloves removal \\
\hline 10) Put on the protective visor & 10) Headgear removal \\
\hline 11) Put on the second pair of surgical gloves. & 11) Filter area exit stepping on a carpet soaked in sodium hypochlorite \\
\hline
\end{tabular}


of authors, is important in the economy of personnel safety and was managed according to the model of the "Organizational Procedures of the Cotugno Hospital", a regional Italian reference center for infectious and contagious diseases.

After completing the dressing phase, operators enter the filter area and then into the negative pressure isolation room where videobronchoscopy is performed.

Eighty videobronchoscopic maneuvers were performed in sedated, intubated and apnea patients, in order to avoid dispersion of aerosolized infected particles. After appropriate pre-oxygenation with pure oxygen for at least 5-10 min, interrupting the flow of air in the ventilation circuit and disconnecting the patient from the upstream HME filter. At this point, after making a small cross incision on the silicone membrane at the top of the mount catheter, the video bronchoscope was introduced into the endotracheal tube and the procedure began. Bronchoscopies were performed as quickly as possible to avoid episodes of severe desaturation $\left(\mathrm{SpO}_{2}\right.$ $<80 \%$ ), in these cases the patient was promptly reconnected to the mechanical ventilator and actively ventilated in pure oxygen until respiratory equilibrium was reached. The mean procedure time was 3-8 minutes.

Seven videobronchoscopic maneuvers were performed in COVID19 patients treated with non-invasive mechanical ventilation (NIV) using a total-face mask, connected to respirator through an HME filter without interrupting the ventilation flow and introducing the bronchoscope through the frontal entry hole covered by a protective silicone membrane.

All bronchoscopies were performed as quickly as possible with disposable single-use bronchoscopes $\left(\mathrm{Ambu}^{\circledR} \mathrm{aScope}^{\mathrm{TM}}\right.$ ) (Figure 3 ), with a probe diameter of $5.0 \mathrm{~mm}$ and a $2.2 \mathrm{~mm}$ working channel connected to the suction circuit and equipped with a dedicated monitor. At the end of the procedure, the operators returned separately to the filter area and under the supervision of another operator at a distance of at least 1 meter, the undressing procedure was carried out (Table 2).

\section{Discussion and Conclusions}

In this work, authors shared the clinical experience acquired in centers with expertise in clinical management of infectious diseases, on WHEN, HOW and WHY a series of 87 bronchoscopies have been performed in suspected or confirmed COVID19 patients. The description of all the details presented in this work represents in the opinion of authors a mine of useful information from which it is possible to obtain practical suggestions and indications on HOW, WHEN and WHY bronchoscopy should be performed in patients affected from complex and contagious infectious diseases such as COVID19.

Furthermore, the clinical experience acquired according to the guidelines of international scientific societies [5-8], allows us to state that bronchoscopy should not be performed in COVID-19 patients with severe respiratory insufficiency in NIV, as this procedure can frequently be responsible of the rapid worsening of symptoms which in many cases can accelerate intubation [8] as well as expose healthcare personnel to a high risk of contagion due to the dispersion of aerosolized particles [5-7]. The evidence collected in this work also testifies that in COVID-19 patients, with severe respiratory insufficiency, the rapid increase in pulmonary resistance is rarely associated with the presence of secretions or clots but more frequently can be linked to pulmonary thromboembolism phenomena. The data disclosed in this work also allow us to state that in intubated COVID-19 patients, subjected to anticoagulant and antiplatelet therapy, the presence of haemorrhage from alveolar damage with the finding of live blood and clots in the respiratory tract is quite frequent and that this clinical objectivity it is generally associated with a poor prognosis. Furthermore, in COVID-19 patients, in our experience, bronchoscopy has proved to be a useful and effective tool for the repositioning of the orotracheal tube and for the correct packaging of the tracheostomy as well as in the suspicion of tracheal damage and aspiration pneumonia.

Furthermore, authors consider it interesting to point out and underline that, during the era of the COVID-19 pandemic, the application of the organizational model for the execution of bronchoscopies implemented and proposed in this paper, allowed such a level of protection that none of the 115 health workers, distributed in the operating units directly involved in the bronchoscopy procedures, was infected.

\section{References}

1. Loeffelholz MJ, Tang YW. Laboratory diagnosis of emerging human coronavirus infections - the state of the art. Emerg Microbes Infect 2020;9:747-56.

2. Verbeek JH, Rajamaki B, Ijaz S, et al. Personal protective equipment for preventing highly infectious diseases due to exposure to contaminated body fluids in healthcare staff Cochrane Database Syst Rev 2020;4:CD011621.

3. Pritchett MA, Oberg CL, Belanger A, et al. Society for Advanced Bronchoscopy Consensus Statement and Guidelines for bronchoscopy and airway management amid the COVID19 pandemic. J Thorac Dis 2020;12:1781-8.

4. Wahidi MM, Lamb C, Murgu S, et al. American Association for Bronchology and Interventional Pulmonology (AABIP) statement on the use of bronchoscopy and respiratory specimen collection in patients with suspected or confirmed COVID-19 infection. J Broncholgy Interv Pulmonol 2020;27:e52-4.

5. Centers for Disease Control and Prevention. Interim U.S. guidance for risk assessment and public health management of healthcare personnel with potential exposure in a healthcare setting to patients with coronavirus disease (COVID19). 2020. Available from: https://www.cdc.gov/coronavirus/2019ncov/hcp/guidance-risk-assesment-hcp.html

6. Group of Interventional Respiratory Medicine, Chinese Thoracic Society. [Expert consensus for bronchoscopy during the epidemic of 2019 novel coronavirus infection (Trial version)]. Zhonghua Jie He He Hu Xi Za Zhi 2020;43:199-202.

7. Gasparini G, Failla G, Agrus AS, Corcione N. [Ruolo e modalità di esecuzione della broncoscopia nella pandemia da COVID 19].[Article in Italian].Position paper AIPONET 17.04.2020. Available from: http:/www.aiponet.it/news/speciale-covid19/2456-ruolo-e-modalita-di-esecuzione-della-broncoscopianella-pandemia-da-covid-19-position-paper.html

8. Darwiche K, Ross B, Gesierich W, et al. [Recommendations for performing bronchoscopy in times of the COVID-19 pandemic].[Article in German]. Pneumologie 2020;74:260-2.

9. Mecham JC, Thomas OJ, Pirgousis P, Janus JR. Utility of tracheostomy in patients with COVID-19 and other special considerations. Laryngoscope 2020;130:2546-9. 